\title{
Effects of Preoperative Tricuspid Valve Diameters on Early Postoperative Surgical Outcomes in Patients Undergoing Tricuspid Valve Surgery
}

\author{
Fatih Ada, MD,${ }^{1}$ Kemalettin Ucanok, $M D,{ }^{2}$ Ahmet Ruchan Akar, MD,${ }^{2}$ Mustafa Bahadir Inan, MD, ${ }^{2}$ \\ Serkan Durdu, MD, ${ }^{2}$ Mustafa Sirlak, MD,${ }^{2}$ Levent Yazicioglu, MD, ${ }^{2}$ Adnan Uysalel, MD,${ }^{2}$ \\ Sadik Eryilmaz, $M^{2}$ \\ ${ }^{1}$ Department of Cardiovascular Surgery, Sivas Numune Hospital, Sivas; ${ }^{2}$ Department of Cardiovascular Surgery, Ankara \\ University School of Medicine, Ankara, Turkey
}

\section{ABSTRACT}

Background: Many of the previous studies on tricuspid valve surgery were on the materials that were used and the advantages and disadvantages of them. In this study, effects of preoperative tricuspid valve diameter on early postoperative outcomes were investigated.

Methods: A total of 43 patients who underwent tricuspid valve repair surgery with the ring between the years 2012-2014 were included in this study. Tricuspid valve diameters and other cardiac functions of patients undergoing tricuspid intervention were evaluated with transthoracic echocardiography.

Patients included in this study were divided into 2 groups: those with minimal, minimal-to-1st degree and 1stdegree tricuspid valve regurgitation found on thoracic echocardiography in the early postoperative period were considered as having a successful tricuspid repair (Group 1). Those with 1st-2nd degree and higher degrees of tricuspid regurgitation were considered as having an unsuccessful tricuspid repair (Group 2).

The relationship between tricuspid valve dimensions and early tricuspid valve regurgitation was assessed with the help of preoperative, intraoperative, and postoperative data.

Results: Thirty patients (Group 1) were found to have a successful tricuspid valve repair in the postoperative period. The mean annulus diameter of the tricuspid valve at enddiastole in patients from Group 1 was similar to Group 2 $(4.24 \pm 0.44 \mathrm{~cm}$ versus $3.99 \pm 0.40 ; P=.080)$. Also, tricuspid valve end-systolic diameter in Group 1 was similar to patients in Group $2(3.59 \pm 0.38 \mathrm{~cm}$ versus $3.42 \pm 0.33 \mathrm{~cm}$; $P=.151)$.

Conclusion: A direct relationship was not found between tricuspid valve diameter and post-operative development of moderate to severe regurgitation in tricuspid valve surgery in this study.

Received May 24, 2017; received in revised form fune 9, 2017; accepted fune 15, 2017.

Correspondence: Fatih Ada, MD, Sivas Numune Hospital, Department of Cardiovascular Surgery, Yeşilyurt, Şifa Cd. No: 4, 58060 Sivas Merkez/ Sivas, Turkey; +9-0346-444-44-58; fax: +9-0346-223-95-30 (e-mail: drfatibada@gmail.com).

\section{INTRODUCTION}

After Kay et al described Kay posterior leaflet plication in 1965, tricuspid valve surgery showed a rapid development [Kay 1965]. As clinical findings in other cardiac valve disorders are more prominent, advances in tricuspid valve surgery are more recent in comparison to other valve surgeries. There are many repair methods in addition to tricuspid valve replacement in tricuspid valve surgery, a few of which are comissurotomy, suture annuloplasty, and ring annuloplasty. The main aim in surgical repair methods is to enable leaflet coaptation by decreasing annulus dimensions and repairing leaflet damage and annular dilation if necessary, thus minimizing valvular dysfunction. All of these methods have different advantages and disadvantages. In this study, the relationship between preoperative tricuspid valve dimensions and early postoperative outcomes after tricuspid valve repair surgery was investigated.

\section{PATIENTS AND METHODS}

43 patients who underwent tricuspid valve repair with ring in the Department of Cardiovascular Surgery of Ankara University Medical School between the years 2012-2014 were included in this study.

Patient data was obtained from their charts and preoperative investigations, and the control data was obtained from transthoracic echocardiography on the 1st postoperative day.

Echocardiographic measurements and the degree of tricuspid valve regurgitation were assessed according to

Table 1. Preoperative Grades of Regurgitation in Patients

\begin{tabular}{lcc}
\hline & Group 1 & Group 2 \\
\hline $2^{\circ} \mathrm{TR}, \mathrm{n}(\%)$ & $9(30)$ & $4(30.7)$ \\
$2-3^{\circ} \mathrm{TR}, \mathrm{n}(\%)$ & $12(40)$ & $5(38.4)$ \\
$3^{\circ} \mathrm{TR}, \mathrm{n}(\%)$ & $6(20)$ & $2(15.3)$ \\
$3-4^{\circ} \mathrm{TR}, \mathrm{n}(\%)$ & $3(10)$ & $2(15.3)$ \\
\hline
\end{tabular}

TR indicates tricuspid valve regurgitation. 
Table 2. Preoperative Demographic Data of Patients

\begin{tabular}{lccc}
\hline & Group 1 & Group 2 & $P$ \\
\hline Age, $y$ & $55.83 \pm 15.9$ & $57.0 \pm 12.5$ & .805 \\
Sex, $\mathrm{n}$ & & & \\
$\quad$ Male & 11 & 5 & .930 \\
$\quad$ Female & 19 & 8 & .945 \\
Hypertension, $\mathrm{n}$ & 6 & 3 & .844 \\
Diabetes mellitus, $\mathrm{n}$ & 4 & 2 & .872 \\
COPD, $\mathrm{n}$ & 4 & 3 & .513 \\
Nephropathy, $\mathrm{n}$ & 1 & 1 & .603 \\
NYHA Classification, $\mathrm{n}$ & & & \\
$\quad$ Class I & 8 & 2 & .433 \\
Class II & 10 & 3 & .546 \\
$\quad$ Class III & 8 & 3 & .826 \\
Class IV & 4 & 5 & .175 \\
Previous cardiac surgery, $\mathrm{n}$ & 3 & 0 & - \\
CVA history, $\mathrm{n}$ & 0 & 0 & - \\
Hepatic dysfunction, $\mathrm{n}$ & 1 & 2 & .290 \\
Smoking, $\mathrm{n}$ & 12 & 3 & .337 \\
BSA & $1.87 \pm 0.21$ & $1.79 \pm 0.22$ & .279 \\
\hline
\end{tabular}

COPD indicates chronic obstructive pulmonary disease; NYHA, New York Heart Association; CVA, cerebrovascular accident; BSA, body surface area.

European Society of Cardiology and American Heart Association guidelines [Lang 2006; Lang 2015]. Measurements of tricuspid valve diameter were done commissure between anterior and septal leaflets to the commissure between the anterior and posterior leaflets, at its widest dimension [Dreyfus 2005]. The degrees of regurgitation of patients in the preoperative period were evaluated by echocardiography as 2 nd degree and more severe and clinically according to the presence or absence of symptoms (Table 1).

The degree of tricuspid valve regurgitation (TR), tricuspid valve systolic and diastolic annulus diameter measurements, left atrial diameter, pulmonary arterial pressure, other valve functions and ejection fractions were evaluated preoperatively.

Three patients had previous operation history in Group 1. There was no previous operation history in Group 2.

Tricuspid ring annuloplasty (TRA, with Medtronic rings of different sizes) procedure was administered as tricuspid valve surgery in all patients included in this study. Tricuspid valve regurgitation etiology was not considered in the study patients.

The patients included in this study were divided into two groups. Group 1: patients having a mild (minimal, minimal to 1 st degree, and 1st degree) tricuspid valve regurgitation in transthoracic echocardiography at the early postoperative phase (postoperative 1st day) constituted those having a successful tricuspid repair; Group 2: those having mild to
Table 3. Preoperative Cardiac Data of Patients

\begin{tabular}{lccc}
\hline & Group 1 & Group 2 & $P$ \\
\hline Tricuspid diastolic diameter & $4.24 \pm 0.44$ & $3.99 \pm 0.40$ & .080 \\
Tricuspid systolic diameter & $3.59 \pm 0.38$ & $3.42 \pm 0.33$ & .151 \\
Mitral regurgitation, $\mathrm{n}(\%)$ & $15(50)$ & $10(76.9)$ & .328 \\
Mitral stenosis, $\mathrm{n}(\%)$ & $10(33.3)$ & $7(53.8)$ & .463 \\
Aortic regurgitation, $\mathrm{n}(\%)$ & $2(6.6)$ & $2(15.3)$ & .705 \\
Aortic stenosis, n (\%) & $4(13)$ & 0 & - \\
ASHD, n (\%) & $2(6.6)$ & $2(15.3)$ & .705 \\
Ascending aortic aneurism, n (\%) & $1(3.3)$ & 0 & - \\
DCMP, n (\%) & $2(6.6)$ & 0 & - \\
ICMP, n (\%) & $1(3.3)$ & 0 & - \\
Pulmonary, n (\%) regurgitation & $1(3.3)$ & 0 & - \\
AF, n (\%) & $15(50)$ & $9(69.2)$ & .467 \\
NSR, n (\%) & $15(50)$ & $4(30.8)$ & .338 \\
PAP & $62 \pm 15.2$ & $53 \pm 10.1$ & .061 \\
Previous cardiac surgery, n & & & \\
CABG & 1 & 0 & - \\
TOF & 1 & 0 & - \\
MVR & 1 & 0 & - \\
\hline
\end{tabular}

ASHD indicates atherosclerotic heart disease; DCMP, dilated cardiomyopathy; ICMP, ischemic cardiomyopathy; AF, atrial fibrillation; NSR, normal sinus rhythm; PAP, pulmonary arterial pressure; CABG, aortocoronary bypass grafting; TOF, tetralogy of fallot; MVR, mitral valve replacement.

moderate and more (1st to 2 nd degree, and more severe) tricuspid valve regurgitation constituted patients having an unsuccessful tricuspid repair.

In comparisons of mortality, mortality during hospital stay of patients from both groups were considered.

\section{Statistical Analysis}

SPSS 15.0 and minitab package programs were used in the statistical analysis in this study. Mean \pm standard deviation [median (minimal - maximal)] was calculated for parameters obtained by measurements as descriptive statistics, and frequency (percent) was calculated for parameters obtained by counting. Mann-Whitney $\mathrm{U}$ test was used in examining the differences between variables of two independent groups obtained by measurement, and Pearson chi-square test was used in examining the differences between variables obtained by counting. $P<.05$ was considered statistically significant. Table values were tested at $95 \%$ confidence intervals.

\section{RESULTS}

\section{Preoperative Findings}

There were no differences between the two groups in terms of age, sex, and BSA (Table 2). The rates of preoperative atrial 
Table 4. Surgical Procedures Administered to Study Patients

\begin{tabular}{lccc}
\hline Surgical Procedure, $n$ & Group 1 & Group 2 & $P$ \\
\hline MVR+TRA & 5 & 2 & .923 \\
MVR+TRA+ASD repair & 1 & 0 & - \\
MVR+TRA+RFA & 7 & 5 & .434 \\
MVR+TRA+KRA & 2 & 0 & - \\
MRA+TRA & 1 & 1 & .603 \\
MRA+AVR+TRA & 1 & 0 & - \\
MRA+AVR+TRA+RFA & 0 & 1 & - \\
AVR+MVR+TRA & 1 & 1 & .603 \\
AVR+MVR+TRA+RFA & 1 & 0 & - \\
AVR+TRA & 2 & 0 & - \\
CABG+MVR+TRA & 2 & 0 & - \\
CABG+MVR+TRA+RFA & 2 & 1 & .909 \\
CABG+TRA & 0 & 1 & - \\
CABG+TRA+RFA & 0 & 1 & - \\
PVR+TRA & 1 & 0 & - \\
LVAD+TRA & 2 & 0 & - \\
LVAD+TRA+RFA & 1 & 0 & - \\
Bentall+MVR+TRA+RFA & 1 & 0 & - \\
\hline
\end{tabular}

MVR indicates mitral valve replacement; TRA, tricuspid ring annuloplasty; $A S D$, atrial septal defect; RFA, radiofrequency ablation; CRA, crioablation; AVR, aortic valve replacement; CABG, aortocoronary bypass grafting; PVR, pulmonary valve replacement; LVAD, left ventricular assist device.

fibrillation, non-tricuspid cardiac valve disease, and mean pulmonary arterial pressures of the two groups were similar (Table 3).

The mean ejection fraction $(\mathrm{EF})$ values of patients in Group $1(49.6 \% \pm 14.8$ versus $52.2 \% \pm 6.3 ; P>.05)$ and left atrial diameters $(5.37 \pm 0.93$ versus $5.53 \pm 0.93 ; P>.05)$ were similar to those of patients in Group 2.

All patients had tricuspid regurgitation. There were no patients with tricuspid stenosis in either group.

\section{Intraoperative Findings}

All patients in both groups underwent a combined surgical procedure, which is presented in Table 4.

In Group 1 in which tricuspid valve diameters were larger, size 32 and bigger rings constituted $63 \%$ of all rings that were used, while this size was used in only $38 \%$ of patients in the Group 2 (Table 6).

\section{Postoperative Findings}

Tricuspid valve repair was considered to be successful in a total of 30 patients. Postoperative echocardiographic findings were classified in 4 groups, independent of successful or unsuccessful results, including minimal TR (min TR), minimal-to-1st degree TR, 1st TR, 1st-2nd degree and higher TR.
Table 5. Early Postoperative Echocardiographic Findings

\begin{tabular}{lccc}
\hline & Group 1 & Group 2 & Mean diameter \\
\hline Minimal TR, n (\%) & $7(16.2)$ & $0(0)$ & $4.22 \pm 0.50 \mathrm{~cm}$ \\
Minimal-1 TR, n (\%) & $10(23.2)$ & $0(0)$ & $4.17 \pm 0.55 \mathrm{~cm}$ \\
1 TR, n (\%) & $13(30.2)$ & $0(0)$ & $4.31 \pm 0.33 \mathrm{~cm}$ \\
1-2 TR and higher n (\%) & $0(0)$ & $13(30.2)$ & $3.99 \pm 0.40 \mathrm{~cm}$ \\
\hline
\end{tabular}

TR indicates tricuspid valve regurgitation.

The number of patients with minimal TR was 7 (16.2\%) with mean diastolic diameter $4.22 \pm 0.50 \mathrm{~cm}$; number of patients with minimal to 1 st degree TR was $10(23.2 \%)$ with mean diastolic diameter $4.17 \pm 0.55 \mathrm{~cm}$; the number of patients with 1st degree TR was 13 (30.2\%) with mean diastolic diameter $4.31 \pm 0.33 \mathrm{~cm}$; the number of patients with $1-2 \mathrm{TR}$ was 13 $(30.2 \%)$ with mean diameter $3.99 \pm 0.40 \mathrm{~cm}$ (Table 5). Ablation was performed in $14(93.3 \%)$ of 15 patients with atrial fibrillation (AF) in Group 1. Ten patient's (71.4\%) rhythm was normal sinus and $4(28.6 \%)$ patient's rhythm was $\mathrm{AF}$ in the postoperative period. In Group 1, preoperative pacemaker was present in 2 patients who underwent LVAD + TRA operation. In addition, postoperative atrioventricular (AV) block developed in $3(10 \%)$ patients in Group 1. A pacemaker was given to 1 of these patients because of permanent $\mathrm{AV}$ block. Ablation was performed in $8(88.8 \%)$ of 9 patients with $\mathrm{AF}$ in Group 2. Six patient's (75\%) rhythm was normal sinus and $2(25 \%)$ patient's rhythm was AF in the postoperative period. In Group 2, no patients had a preoperative pacemaker. Postoperative AV block developed in 1 patient but no permanent pacemaker was needed in Group 2.

\section{DISCUSSION}

Primary isolated tricuspid regurgitation is a rare condition, and the most frequent pathology that we encountered was functional-type tricuspid regurgitation, which was seen at a rate of 70-85\% [Antunes 2007]. This develops due to insufficient coaptation of leaflets, due to derangement in systolic annulus contraction and tricuspid annulus dilation as a result of increased pulmonary pressure and increased right ventricular pressures, and it may be secondary to left heart valvular disease [John 2009]. Derangement of right ventricular geometry, changes in shape and dimensions of the tricuspid valve and annulus, and changes in the length of papillary muscle are the most important factors in tricuspid regurgitation which develop secondary to left heart failure. The remaining $15-30 \%$ of patients are those that are seen in conditions that the tricuspid valve is directly affected by rheumatic disease or organic causes. Mild to moderate TR may regress after treatment of left heart valvular disease [Nurözler 2000]. But surgical treatment is required for moderate to severe TR, as it increases postoperative morbidity and mortality. Tricuspid valve regurgitation that is present before mitral valve surgery or persists after surgery is a predictor of poor prognosis. 
Table 6. Ring Size of Tricuspid Valve Repair

\begin{tabular}{|c|c|c|c|c|c|}
\hline Group 1 & $2(6.6)$ & $9(30)$ & $15(50)$ & $3(10)$ & $1(3.3)$ \\
\hline
\end{tabular}

Prolapse of tricuspid valve is present in $30-40 \%$ of cases with mitral valve prolapse. Tricuspid regurgitation is mild in these cases. A mild dilation of the right atrium, tricuspid regurgitation of first or second degree are known to regress with a decrease in pulmonary artery pressure. In cases in which moderate to severe tricuspid regurgitation is present, tricuspid annuloplasty with mitral repair is recommended [Shiran 2009].

Repair techniques, which have developed since the beginning of 1960s, show variability today, with their advantages and disadvantages. Although interventions to the leaflets and chords in repair of the tricuspid valve were done, the importance of increasing annulus diameter in the pathophysiology of regurgitation and decreasing annulus diameter has become the gold standard in tricuspid repairs.

Ring annuloplasty was performed in all patients who were included in our study.

There are few studies on preoperative valve diameter measurements in tricuspid valve surgery. In our study, the effects of preoperative tricuspid valve diameter measurements on early echocardiographic regurgitation findings were investigated.

In the study by Dreyfus et al, the distance between the anterior and septal leaflets of the tricuspid valve were measured intraoperatively by means of a ruler and tricuspid valve repair was done without consideration of the degree of regurgitation when the measured diameter was over $70 \mathrm{~mm}$. While no significant differences were observed between the group undergoing mitral valve surgery whose intraoperative valve diameter was below $70 \mathrm{~mm}$ and patients who had undergone tricuspid valve repair, in the longer-term follow-up, severe tricuspid regurgitation was observed in only those patients in whom interventions to the mitral valve were done [Dreyfus 2005].

Colombo et al evaluated preoperative tricuspid valve diameters in tricuspid regurgitation secondary to the mitral valve and found the mean tricuspid annulus diameter as $40.59 \mathrm{~mm}$. This study included 50 patients who had tricuspid regurgitation secondary to mitral regurgitation and who had undergone surgery. The mean tricuspid annulus diameter was found to be $40.59 \mathrm{~mm}(>21 \mathrm{~mm} / \mathrm{m} 2)$ and surgical repair was found to have positive effects on postoperative late-phase cardiac functions. These tricuspid valve diameter measurements are similar to those that were found in our study [Colombo 2001].

In the study by Shahzad et al, functional tricuspid regurgitation was divided into 3 phases, and perfect outcomes from tricuspid repair were reported in the first two phases, as the problem was only tricuspid valve annulus dilation, without tricuspid valve tethering. On the other hand, the problem was not only annular dilation in the 3 rd phase, but included valvular tethering, and annuloplasty alone did not provide satisfactory outcomes [Shahzad 2010].
According to the ESC guidelines published in 2007, tricuspid valve repair was recommended for patients who would undergo left side valvular surgery who had moderate organic tricuspid regurgitation, and also to patients who would undergo left side valvular surgery, had tricuspid annulus diameter over $40 \mathrm{~mm}$, and who had moderate secondary tricuspid regurgitation [Dreyfus 2009].

In another study by Ubaga et al, maximal early systolic diameter was found to be $21 \pm 2 \mathrm{~mm}$ in the group without cardiac problems in calculations of division of body surface area to tricuspid valve systolic diameters, $24 \pm 2 \mathrm{~mm}$ in those having other valvular problems without tricuspid regurgitation, $31 \pm 4 \mathrm{~cm}$ in patients having mild tricuspid regurgitation, and $37 \pm 4 \mathrm{~mm}$ in patients with moderate to serious tricuspid regurgitation [Ubaga 1983].

The values found in our study are similar to those found in the study by Ubaga in terms of presence of other valvular problems and presence of at least 2 nd degree regurgitation in the preoperative patients (mean diameter in Group 1 was $3.59 \pm 0.38 \mathrm{~cm}$ and $3.42 \pm 0.33 \mathrm{~cm}$ in Group 2).

Although there are no studies in the medical literature on the relationship between measurements of tricuspid valve diameters and mortality, in our study mortality was $6.6 \%$ in Group 1 and 7.6\% in Group 2. There were no significant differences between these two groups in terms of the degree of early phase regurgitation and mortality.

\section{Study Limitations}

A statistically significant association was not found between tricuspid valve dimensions and postoperative early phase moderate to severe tricuspid valve regurgitation. But explanation of tricuspid valve regurgitation developing in the early postoperative period by only valve measurements is insufficient. The reason is that it is affected by the complexity of surgical procedure used, tricuspid repair with or without the use of cross clamp, the duration of time between phase of disease and surgical intervention, etiology of tricuspid valve regurgitation, and procedural differences of surgeons doing the operation. Also, the small sample size in this study may have had an effect on results. Studies with a larger number of patients and with specific groups may enable a clearer observation of outcomes.

\section{Conclusion}

In light of this information, a direct relationship between preoperative valve diameter and postoperative mediumto-severe regurgitation was not found in tricuspid valve surgery. At the same time, repairing of corrupted tricuspid valve's geometry seems as indifferent on large diameter or small diameter tricuspid valves on early postoperative period; 
however, midterm or long-term results may be much different on the follow-ups. But larger scale studies are required for investigations on the effects of tricuspid valve diameter on postoperative outcomes in certain patient subgroups undergoing certain surgical procedures.

\section{ACKNOWLEDGEMENT}

Thank you to Saadet Aslan for echocardiographic measurements and patient data.

\section{REFERENCES}

Antunes J, Barlow JB. 2007. Management of tricuspid valve regurgitation. Manuel Heart 93:271-6.

Colombo T, Russo C, Ciliberto GR, Lanfranconi M, Bruschi G, et al. 2001. Tricuspid regurgitation secondary to mitral valve disease: tricuspid annulus function as guide to tricuspid valve repair. Vascular 9:369-77.

Desai RR, Abello LMV, Klein AL, et al. 2013. Tricuspid regurgitation and right ventricular function after mitral valve surgery with or without concomitant tricuspid valve procedure. J Thorac Cardiovasc Surg 146:1126-32

Dreyfus GD, Corbi PJ, John Chan KM, Toufan B. 2005. Secondary tricuspid regurgitation or dilatation: which should be the criteria for surgical repair? Ann Thorac Surg 79:127-32.
Dreyfus GD, John Chan KM. 2009. Functional regurgitation: a more complex entity than it appears. Heart 95:868-9.

John C, Mustafa Z, Emre A, Prakash PP. 2009. Tricuspid valve disease: pathophysiology and optimal management. Cardio Dis 51:482-6.

Kay JH, Maselli CG, Tusju HK. 1965. Surgical treatment of tricuspid insufficiency. Ann Surg 162:53-8.

Lang RM, Bierig M, Devereux RB, et al. 2006. Recommendations for chamber quantification. Eur J Echocardiogr 7:79-108.

Lang RM, Bierig M, Devereux RB, et al. 2015. Recommendations for chamber quantification: a report from the American Society of Echocardiography's Guidelines and Standards Committee and the Chamber Quantification Writing Group, developed in conjunction with the European Association of Echocardiography, a branch of the European Society of Cardiology. J Am Soc Echocardiograph 18:1440-63

Nurözler F. 2000. Tricuspid kapak tamirinde cosgrove-edwards anuloplasti ringi kullanimi. Turk gogus kalp dama 8:576-8.

Shahzad RG, Dreyfus GD. 2010. Basis for intervention on functional tricuspid regurgitation. Seminars in Thorac Cardiovasc Surg 22:79-83.

Shiran A, Sagie A. 2009. Tricuspid regurgitation in mitral valve disease: incidence, prognostic implications, mechanism, and management. J Am Coll Cardiol 53:401-8.

Ubago JL, Figueroa A, Ochoteco A. 1983. Analysis of the amount of tricuspid valve annular dilatation required to produce functional tricuspid regurgitation. Am J Cardiol 52:155-8. 